\title{
TOWARDS A SOLUTION FOR INTEROPERABILITY OF SMARTHOMES DEVICES
}

\author{
Héldon José O. Albuquerque ${ }^{1}$ and Gibeon S. Aquino Junior ${ }^{1}$ \\ ${ }^{1}$ Department of Informatics and Applied Mathematics-DIMAP Federal \\ University of Rio Grande do Norte, Natal, Brazil
}

\begin{abstract}
During the recent years, we can observe how mobile devices entered in the lives of people, becoming their main personal assistants and helping in various daily tasks. However not just mobile devices have evolved. And others electronic devices we use every day also experienced changes that have become smarter as the example of home devices. All these devices interconnected in the same environment or the same network, making use of services and exchange information with other devices, characterize a smart environment. smarthomes are special class of such environments and, increasingly, has become a scene with a variety of heterogeneous devices. However, due to the rapid progress of technology and the rise of a large number of heterogeneous devices, a variety of independent communication protocols were created, establishing a complex scenario to ensure interoperability between them. In this context, the main objective of this paper is to analyze the state of the art with an emphasis on the interoperability of current Mobile Devices with other digital devices present in a smarthome. Thus, it will be defined which protocols are currently most commonly used for this purpose, what are the current ongoing projects, what are the limitations of the solutions found in the research and, finally, it will be proposed an alternative solution for interoperability between the devices at a smarthome..
\end{abstract}

\section{KEYWORDS}

smarthome, systematic review, proxy, connectivity, communication protocol, interoperability, mobile devices.

\section{INTRODUCTION}

In 2005, [15] assumed that mobile computing would, in a not so distant future, replace computing based on "desktop computers", which were, on the other hand, already an improvement compared to the "mainframe computers". This supposition, according to [11, 13, 18 , actually became true nowadays. Furthermore, they indicate that ubiquitous computing is the natural successor of mobile computing and, therefore, a reality.

Mobile devices began to emerge in the 80s [3], however, it was only in early 2000 that the first devices with a considerable processing capacity appeared [7]. Among these were smart-phones and tablets. These devices gained more notability than personal computers because of the mobility that they allow and provide.

Dhinaharan Nagamalai et al. (Eds) : CSE, DBDM, CCNET, AIFL, SCOM, CICS, CSIP- 2014

pp. 261-272, 2014. (C) CS \& IT-CSCP 2014

DOI : $10.5121 /$ csit.2014.4423 
Over the past few years, mobile devices have become essential items in daily life. They became everybody's main personal assistants, used as a tool to aid in various tasks of day to day life.

However, mobile devices are not the only ones that have progressed. Kaed et al [11], points out that other devices and electronics that were not mobile or portable underwent changes that made them more "smart". An example of such are appliances like televisions that display internet content, which have the ability to connect with other devices to allow audio and video transmissions. There are refrigerators that make shopping lists, air conditioners and lights that are controlled over the Internet, among other examples of smart devices that function without mobility. All of these devices interconnected in the same environment, or on the same network, and inter-acting with other devices features an intelligent environment [5].

In September 2003, "Housing Learning \& Improvement Network" created a project called DTI Smart Homes Project, offered by INTERLEK. This work is the first definition of an intelligent environment. In particular, there is a smarthome, also known as smart house, automated home or home automation [20].

A smarthome is an environment with a diversity of devices that use heterogeneous communication protocols, and in which the interoperability between them is a critical part to their contribution, but also one of the main problems found [1]-[5]-[18]. Most devices are independent and offer specific protocols so that they can be discovered in a home network.

In this context, the main objective of this paper is to analyze the state of the art with an emphasis on the interoperability of current Mobile Devices with other digital devices present in a smarthome. Thus, it will be defined which protocols are currently most commonly used for this purpose, what are the current ongoing projects, what are the limitations of the solutions found in the research and, finally, it will be proposed an alternative solution for interoperability between the devices at a smarthome.

The remainder of this paper is organized as follows: Section 2 defines the systematic review to find out the state of the art protocols involving this context smarthome. Section 3 defines the work related to the objective of this research. Section 4 highlights potential problems with the main conclusions. In Section 5, a generic suggestion will be done to effectively solve the problem that was addressed. Finally, in Section 6, the final considerations resulting from this research will be presented.

\section{SYSTEMATIC REVIEW}

To perform the investigation proposed in this work it was used as methodology, a systematic review, in order to ensure a theoretical and practical background in the state of art of the proposed theme. Second [14], "a systematic review is a form to identify, evaluate and interpret all the available and relevant research to a specific research question, or topic area, or interest phenomenon".

The main goal of a systematic review is to integrate the information of a number of studies performed separately about a specific topic or issue, with the purpose of revelling conflicting and/ or coincident results, as well as to identify themes that need evidences and help in the orientation to investigations for future work [10]. 


\subsection{Systematic Review Protocol}

The Systematic Review Protocol defines the rules and steps to be adopted to the performance of the research in the state of the art. In the following subsections the principal steps that constitute the protocol of elaboration of this systematic review are briefly described.

\subsubsection{Objectives of the study and Research questions}

Based on the proposed theme, the objective for this systematic review is to perform an analysis of the state of the art of interoperability of the current Mobile Devices with the digital devices of a smarthome. In particular, it is intended to investigate the connectivity protocols and technologies and the developed and proposed solutions in this environment.

Based on the established objective and following the recommendations approached in [14], it was prepared the following research questions:

- RQ1 - What are the protocols and technologies that permit the connectivity among the current Mobile Devices and the devices of a smarthome?

\subsubsection{Search and selection of the articles}

Approaching the keywords contained in the research questions, contextualized to the ambience of mobile computing, Ubiquitous and Pervasive, it was defined the following string of search.

- (((Ubiquitous OR Pervasive) AND Computing) OR Internet of Things) AND ((Mobile Device) AND (Interoperability OR Connectivity OR Communication OR integration ) AND (smarthome))

Articles that are available only on a paid form, along with articles that have more than ten years from the date of publication, were discarded. At last, the search vehicles used for this research were:

- ACM Digital Library;

- IEEEXplore Digital Library

\subsubsection{Search and selection of the articles}

According to [14], the form to evaluate and extract data from the research is defined in the Execution phase. In this one, the tests of execution are performed, that is, some of the publications based on the selection procedure of the review protocol are chosen. During this step, the primary studies are identified, selected and evaluated regarding the selection procedure.

Table I exposes the quantity of references retrieved according to the search vehicles, the quantity of articles selected for the reading and the quantity of articles accepted regarding the selection procedure. To avoid an exhaustive analysis e based on the fact that the results are ordered by relevance, the maximum of the first 50 works returned in the search in the vehicles were selected. 
Table 1. Execution Table

\begin{tabular}{|c|c|c|c|}
\hline Vehicle & Returnees & Selected & Accepted \\
\hline ACM & 46 & 46 & 9 \\
\hline IEEE & 97 & 50 & 14 \\
\hline
\end{tabular}

\subsubsection{Information Extraction of the Systematic Review.}

The main goal of the protocols of interconnection in a smarthome is to provide flexibility in information sharing, in a transparent manner among the devices, being mobile or electronic, and especially for the final user [17].

The connectivity protocols facilitate the interaction among devices, electronics and computers, dismissing the problem with the configuration of the entrance of a new component in the ambience and, consequently, freeing the user to do it. Thus, a user without technical knowledge will be able to monitor and control equipment's such as fridges, air conditioner, stove, lights, windows, doors, TVs, ventilators, printers and other computing devices, simply from another device $[4,11,13,7]$.

Due to the accelerated advancement of technologies and the emergence of a great quantity of heterogeneous devices in the same smarthome, a variety of protocols of independent communication were created $[1,11,23]$, establishing a complex scenario of ensuring the interoperability among them.

This section presents the result of the research about the state of the art of the protocols of communication and connectivity in a smarthome, answering the research question RQ1, defined in section 2 .

Table 1 presents the protocols used in the 23 articles analysed. It is noteworthy that, some of these works just mentioned protocols capable to manager devices present in a smarthome, but didn't use them in their researches. For our analyses, we consider only the protocols directly used in the research works.

Table 2. Connectivity Protocols

\begin{tabular}{|c|l|}
\hline Protocols & \multicolumn{1}{|c|}{ References } \\
\hline UPnP & $\begin{array}{l}{[1]-[5]-[6]-[8]-[9]-[11]-[12]-} \\
{[13]-[16][18]-[21]-[23]-[24]}\end{array}$ \\
\hline DLNA & {$[2]-[5]-[8]-[17]-[19]-[21]$} \\
\hline ZigBee & {$[13]-[17]-[18]-[23]-[25]$} \\
\hline DPWS & {$[11]-[12]$} \\
\hline JINI & {$[1]$} \\
\hline Bluetooth SDP & {$[9]$} \\
\hline
\end{tabular}

The Universal Plug and Play (UPnP) was initialized by Microsoft in the year 1999 as a model of network architecture that provides connectivity ad-hoc among devices in a distributed manner, transparent, independent of driver or platform and with no need of any kind of configuration. One of the main characteristics of the UPnP, is the facility to provide services in the network, owning a protocol of device discovery and, consequently, of services. A main limitation of the 
UPnP is that its structure is limited to an only network LAN. Companies as Nokia, Intel and HP, collaborated with this protocol, emerging from this partnership the UPnP Forum ${ }^{1}$.

The Digital Living Network Alliance ${ }^{2}$ (DLNA) is a standard established in 2003 by SONY and adopted by many companies in the industry. It is a connectivity protocol which has as objective to promote the interoperability among equipment's and electronic devices, mobile devices and personal computers. This builds upon the UPnP protocol, leveraging the power of its discovery, entreated, DLNA define particular characteristics for the exclusive use of its protocol.

The ZigBee, is a wireless network protocol developed by Alliance $\mathrm{ZigBe}^{3}$ with techniques of small energy consumption wireless communication and short distance frequency. The main characteristics of ZigBee are the low transmission of data and the low cost of the devices. One of the projects developed by Alliance ZigBee is the ZigBee Home Automation, which offers an interoperability standard for domestic and digital equipment's, and devices with platforms of development, allowing, this way, the integration with devices in other ZigBee networks.

The Device Profile for Web Service (DPWS) emerged in 2004 and was acknowledged and standardized in 2008. Maintained by OASIS ${ }^{4}$, the DPWS is a stack of protocols that define a minimum set of functionalities so that resources limited devices can adopt Web Service (WS) standard. Its main characteristics are the event notification, message exchange, discovery service and description of services, developing mechanisms of high level communication for interoperability among devices present in a smarthome.

The JINI ${ }^{5}$ protocol was created in 1998 and maintained by Sun Microsystem. As well as the protocol UPnP, DPWS and DLNA, the JINI is a protocol that enables devices to connect to each other and share resources. The devices involved can be, from personal computers to mobile and electronic devices. However, it is limited to the JAVA platform, can be used in any mobile device that uses this platform.

At last, the protocol Bluetooth Service Discovery Protocol (BSDP) is used to allow that, devices, through the Bluetooth technology, can discover which services other devices bear or provide. The BSDP has as basis the protocol Service discovery Protocol (SDP), which is the same used by UPnP and DLNA to discover devices in a network. The BSDP is maintained by the organization Bluetooth $\mathrm{SIG}^{6}$.

Some of the analysed articles use a combination of proto-cols in their answers in order to create a middleware among them, as the example of [8]. In this work, it is proposed a module for transparent replication of a DIGITAL TV in networks based on UPnP and DLNA protocols for a smarthome. Devices that endure only the UPnP protocol, can receive information from a DIGITAL TV that only uses the DLNA protocol and vice versa.

As can be seen in the graphic of Figure 1 of the found protocols, the UPnP stands out in relation to the others, in other words, $46 \%$ of the articles prefer to use the UPnP protocol to perform the communication among the Mobiles Devices with the devices in a smarthome. Coming in second the DLNA protocol with $26 \%$, and in third, the protocol ZigBee with $18 \%$.

\footnotetext{
${ }^{1}$ http://www.upnp.org

${ }^{2}$ http://www.dlna.org

${ }^{3}$ http://www.zigbee.org

${ }_{5}^{4}$ www.oasis-open.org

${ }^{5}$ http://www.jini.org

${ }^{6}$ https://www.bluetooth.org
} 


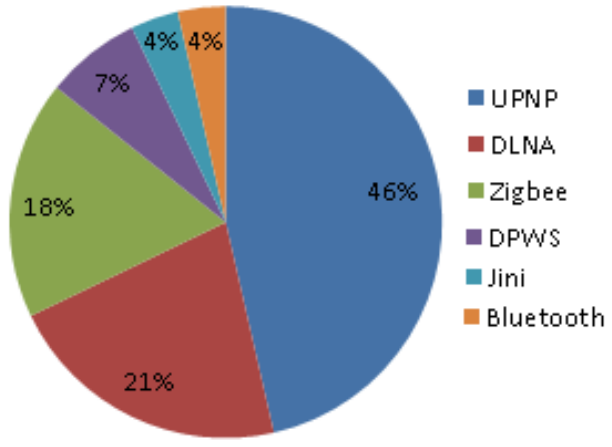

Figure 1. Connectivity Protocols.

The UPnP protocol stands out in the selected works for being an open standard and mainly for being based on the IP protocol, becoming a compatible protocol with any equipment turned to the network connection [23]. It has a light architecture that allows its use either for high technology devices, such as the micro-controllers, being able to be used to perform simple operation of turn on-turn off (Light- UPnP), or perform procedure to make specific decisions (Presence- UPnP) [16]. Besides, it is a protocol that operates using the peer-to-peer technology, not requiring any equipment or technology present among the devices participants to integrate them. At last, one the main reason for its use is that it is protocol based on the Plug and Play model, facilitating the installation and configuration of devices [6].

Examples of other connectivity protocols used in a smarthome, X10 [13], HAVI [22], IGRS [11], Salutation [2], GENA [16], Z-Ware [13], Ninja [1], were also mentioned in the articles, although they were not used with main focus.

\section{RELATED WORKS}

The variety of functionalities desired in a smarthome is increasing the complexity of the systems involved in this area. This complexity has motivated research groups to discover solutions in this context. One of the well mentioned and discussed problem is the one in intercommunication among devices [24]. With the objective of answering the Research Question RQ2 proposed in section 2, the research projects reported in the articles were evaluated.

In [18], it is developed a bridging protocol, for communication among the protocols UPnP and ZigBee. To the development of this bridge, it was also used the COAP protocol (Constrained Application Protocol) which is used in electronic devices. This solution will provide flexibility in communication among devices with the UPnP and ZigBee protocol, making their particularities transparent among them.

[11] has developed the middleware INSIGHT, with capacity to provide the interoperability and services management in a smarthome over the UPnP and DPWS protocols.

In [25], it is presented the InfoPods system. An open architecture composed by a controller based on the ZigBee protocol, allowing that any device which uses this protocol can control the electronic devices belonging to a smarthome.

Besides these ones, other projects were also identified in a direct manner, as the articles were read and mentioned research works that were not present in the articles resulting from the execution of the search String. Among these projects, we can detach the followings: 
The Service Bus Device (SBD) is a middleware which has as function to integrate heterogeneous devices in a Ubiquitous environment. This project was developed in a portable platform with the purpose of being executed in any kind of platform, being mobile or electronic. It has as the main objective to create a communication bus so that the equipment's with specific technologies, like the RFID , Bluetooth, Wi-fi, can offer services in the network. This one uses the DPWS protocol to perform the interoperability among the devices present in the ambience.

The Accessing We-based Applications on Consumer De-vices project (Web4CE) defines a network architecture that allows the devices present in a smarthome to directly access the web, without depending on other technology for that, being possible to access its individual configurations. This project uses the DLNA protocol for the interoperability of its network with other devices.

The MavHome is a project of a smarthome, developed by the Texas University. Its objective in to make the ambiences adaptive, that notices the state of the house with the help of sensors. In it, intelligent devices control the ambience ensuring the user's comfort by the recognition of activity, using video cameras and sensors in general. These devices process data to acknowledge what the users are doing, therefore, can take some initiatives to foresee what the user would do then, helping him somehow and minimizing the effort of the user.

Other proposes, like $[12,17,18,21]$, have as objective to allow that distinct protocols can interconnect to each other in a smarthome, avoiding the particularities of each protocol. The main motivation for the majority of the accepted works in this research is to develop middleware platforms of integration, with the purpose of interconnecting all kinds of devices with different communication technologies in the same ambience.

Although the solution with the use of middleware's being accepted, works like [16, 19, 25] defends a solution with the use of an only connectivity protocol in a smarthome. This approach would decrease the complexity offered to the integration of different kinds of protocols and would be certainly more performativity and economic.

The fact is that in the current estate of technologies development, an integrative solution, based on middleware's, is the faster and flexible answer to ensure the interoperability among the devices. Although, in a long-term vision, the most appropriate way would be that only one standard predominate. This standard would be evolved enough to incorporate the characteristics and advantages of the others, besides being open and maintained by a large number of representatives of industry and academy. Probably, the UPnP is the protocol nearest this reality, but still needs to evolve a lot from the technical point of view to be considered substitute of the others.

\section{Limitations Founding Solutions}

An intelligent environment contains many interconnected heterogeneous devices. They use services or exchange in-formation in a transparent and dynamic way [5]. According to [12] and [17], the ability to be automatically recognized in an environment is fundamental to the achievement of an intelligent environment and is also the most fascinating role of the protocols identified in this paper. Moreover, the diversity of these protocols and technologies that are available nowadays makes the seamless interoperability between devices inhibitive and, thereafter, prevents the full implementation of smart home environments.

As an overview of the architectural behaviour of the integrated protocols in a smarthome, we can identify two common technical features between the protocols. 
First, these protocols use the communication protocol based on TCP/IP, or, in other words, any device that supports a protocol for a smarthome can be integrated using any means of communication, whether it is Ethernet, Wi-Fi, etc. However, it is necessary that the machine is able to acquire an IP address.

Second, the components of a smarthome [11] can be classified in two different ways: controllable devices - those who provide the services that the devices offer; and control point those responsible for using the services provided by the devices.

Also according to [11], other components may arise de-pending on the protocol, but in general, these protocols are based on the client-server concept, where the client is known as Control Point and the server is known as the Device.

Although these protocols have similar high level goals, they are composed of very different architectures. Each machine using the discovery of service will use only one of these protocols; for example, the UPnP control point will only find in a network the UPnP devices, and so it happens for the other protocols. This means that clients and services using different technologies will not be able to cooperate. Since it is likely that several protocols will be widely used in the environment, there is a need for a structure of interoperability that allows clients and services written using different protocols to cooperate.

Until now, it was realized that there are two possibilities to integrate a new device with a protocol not yet supported by the environment, as shown in Figure 2.
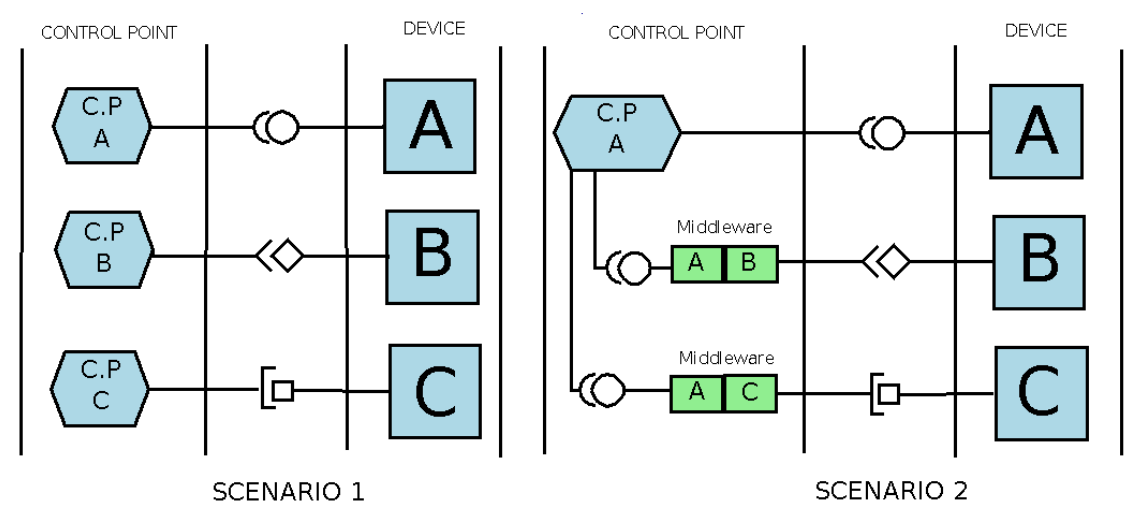

Figure 2. Scenarios insertion of a new protocol

It is observed in Scenario 1 that, in the integration of multiple devices with different protocols in a smarthome, it is necessary to add control points to interact with the services provided by the device. For each device with protocols A, B and C, control points C.P-A, C.P-B and C.P-C must be added to the environment. The main problems related to this scenario is the amount of control points managed by the user, creating a non-scalable solution, since the main goal of a smarthome is to reduce the user's effort in controlling the devices in the environment; and the noninteraction between devices, since each device can only support a single protocol, and these protocols do not recognize each other in the same environment.

In Scenario 2, on the other hand, it is observed that the middleware's are solutions widely used for interoperability between different communication protocols in smarthomes. More concretely, the problem occurs when we add devices with connectivity protocol $\mathrm{B}$ and $\mathrm{C}$ in an environment that supports only protocol A. middleware's must be developed and integrated directly into the 
control point A to interconnect the new protocols on the environment. In this case, we observed as main advantage the minimization of the overhead produced by the conversion of packages between the protocols, since there is more than one layer of middleware on a single control point. In contrast, the existing solutions tend to be intrusive in control points. In other words, to integrate any middleware layer in control points, which would be the most ideal solution for the reuse of existing control points, the direct access to their source code should be allowed. However, many existing solutions do not provide this access to the code.

However, [16] emphasizes that the diversity of middlewares present in a smart environment will provide, at some point, incompatibilities when they interconnect with each other. Moreover, such solutions produce a gradual increase in the complexity of the environment and these solutions found so far are directly linked to a change in the context of the control point.

\section{Proposal for a Generic Solution}

In this section, a proposal of interoperability solution between the protocols of a smarthome will be presented. The proposal is based on the introduction of a proxy service that will allow the interoperability of different protocols without changing any of the existing components in the environment. As can be seen in Figure 3, we will add a physical device to the communication infrastructure, which will make the translation of communication packages between protocols possible.

Thus, the control point A (PC-A) will be able to communicate normally with devices with protocol A and also with the other protocols in the environment through the proxy, without the need of any programmatic modification in its structure.

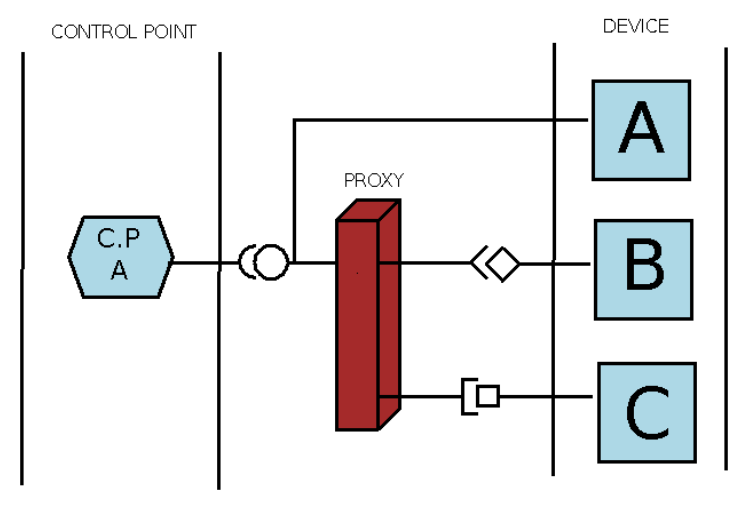

Figure 3. Proposal for the interoperability between the protocols

In this article, we will focus on the interoperability between UPnP and DLNA protocols because they are the most important protocols in the current scenario. Despite the high-level similarities between these protocols, since they both use consolidated and standardized internet protocols such as TCP/IP, HTTP, SOAP and others as their basis, the exchange of information of services provided by devices and the interoperability between them is not simple. By using the same language for data exchange of services provided by devices, each protocol uses an independent XML scheme, in other words, the XML scheme used by each protocol defines different validation rules, this being the main reason for the inability of these protocols to interact with each other. 
As a way to assess the applicability of the proposed solution, a case study was implemented considering a scenario where already developed UPnP Control Points interact with DLNA devices existing in the environment.

This proposal essentially consists of "virtual services" that will be automatically instantiated by the proxy in response to the discovery of DLNA devices in the network. To achieve this goal, we need to "trick" the UPnP Control Point, making it believe that it is interacting with the services provided by an UPnP device, but which are in fact services provided by DLNA devices.

In the scenario above, the proxy creates virtual UPnP devices for each DLNA device found in the environment, recording the services provided by each device as illustrated in Figure 4. For each instance of service supported, virtual de-vices will be responsible to make a bridge between protocols, translating the XML file format of the virtual UPnP device's services to the DLNA and performing the interoperability between the protocols. When a DLNA device is removed from the network, the proxy will automatically destroy the corresponding virtual services.

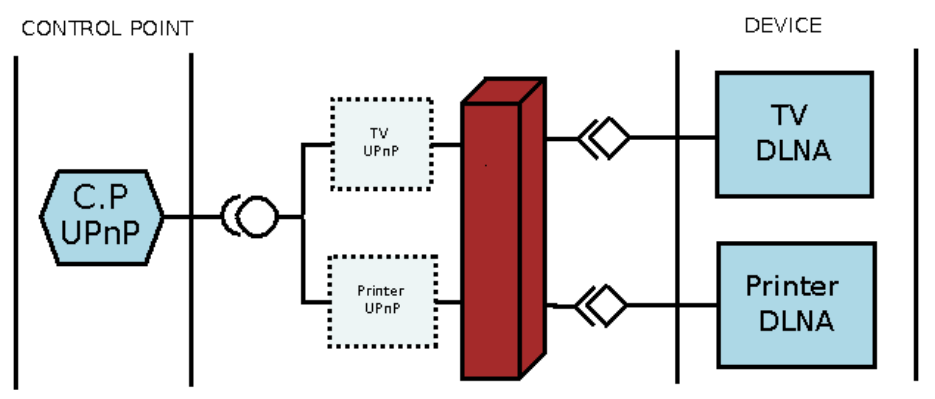

Figure 4. Proxy for interoperability between UPnP and DLNA protocol

To achieve this goal, the proxy architecture is divided into three basic components:

- Discovery Module: Provides basic functions of a DLNA Control Point, like the discovery of the services provided by devices in the environment and detection of change in the values of the state variables of the service provided. The first function is needed to transmit the commands to a DLNA service through the virtual UPnP device, while the second is responsible for updating the values of the variables of the virtual device;

- Analysis Module: Responsible for managing the other modules of the system, managing the services provided by devices, requesting the database of the UPnP devices the most adequate device to perform the translation between protocols, managing the state variables of the services provided by the Discovery Module and by the virtual UPnP devices;

- Virtualization Module: Component used to implement the virtual UPnP devices. This module is also responsible for ensuring that the same device is not duplicated when a state variable of a service is modified.

For each new device in the environment that is not sup-ported by proxy, a modest amount of code must be written to adapt the proxy for its recognition so UPnP/DLNA and its Control Points do not need any modification. One of the main goals of this "proxy" is the reduction of the effort in the use of already developed applications. 
Computer Science \& Information Technology (CS \& IT)

\section{Conclusions}

We present a proposal for the interoperability between the protocols of a smarthome and demonstrate the viability of the proposal by applying it to a context involving DLNA and UPnP protocols. Our proposal will introduce virtual services that allow DLNA customers to interact with UPnP Control Points already developed, aiming at the reuse of existing solutions in the market and, consequently, at a reduction of efforts to create or adapt existing middleware solutions. The efforts of this solution are important because, in the short term, with the lack of both academic and industry initiatives for the development of a standard that involves the field of smart environments, a number of technologies and protocols will compete for space, thus overloading an increasing number of independent protocols in an environment, making them increasingly heterogeneous.

\section{REFERENCES}

[1] Sameh, A.; El-Kharboutly, R., "Modeling Jini-UPnP Bridge using Rapide ADL,"Pervasive Services, 2004. ICPS 2004. IEEE/ACS International Conference on, vol., no., pp.237,237, 19-23 July 2004.

[2] S.V. Anand. A dlna framework for next gen mobile terminals connecting ims networks for humancentered digital home environment. In IP Multimedia Subsystem Architecture and Applications, 2007 International Conference on, pages 1-5, 2007.

[3] Daniel Barbar' a. Mobile computing and databases-a survey. IEEE Trans. on Knowl. and Data Eng., 11(1):108- 117, January 2009.

[4] C. Beckel, H. Serfas, E. Zeeb, G. Moritz, F. Gola-towski, and D. Timmermann. Requirements for smart home applications and realization with ws4d-pipesbox. In Emerging Technologies Factory Automation (ETFA), 2011 IEEE 16th Conference on, pages 1-8, 2011.

[5] Wally Chen, Sy-Yen Kuo, and Han-Chieh Chao. Service integration with upnp agent for an ubiquitous home environment. Information Systems Frontiers, 11(5):483- 490, November 2009.

[6] R. Chowdhury, A. Arjona, J. Lindqvist, and A. Yla-Jaaski. Interconnecting multiple home networks services. In Telecommunications, 2008. ICT 2008. International Conference on, pages 1-7, 2008.

[7] M.N. Cortimiglia, A. Ghezzi, and F. Renga. Mobile applications and their delivery platforms. IT Professional, 13(5):51-56, 2011.

[8] Giliard Brito de Freitas and Cesar Augusto Camillo Teixeira. Ubiquitous services in home networks offered through digital tv. In Proceedings of the 2009 ACM symposium on Applied Computing, SAC '09, pages 1834-1838, New York, NY, USA, 2009. ACM.

[9] A. Delphinanto, A. M J Koonen, M. E. Peeters, and F. T H Den Hartog. Proxying upnp service discovery and access to a non-ip bluetooth network on a mobile phone. In Communications and Vehicular Technology in the Benelux, 2007 14th IEEE Symposium on, pages 1-5, 2007.

[10] O. Dieste, M. Lopez, and F. Ramos. Formalizing a sys-tematic review updating process. In Software Engineering Research, Management and Applications, 2008. SERA '08. Sixth International Conference on, pages 143-150, 2008.

[11] Charbel El Kaed, Antonin Chazalet, Lo“ 1c Petit, Yves Denneulin, Maxime Louvel, and François Gaël Ottogalli. Insight: interoperability and service management for the digital home. In Proceedings of the Middleware 2011 Industry Track Workshop, Middleware '11, pages 3:1- 3:6, New York, NY, USA, 2011. ACM.

[12] Charbel El Kaed, Yves Denneulin, and Francois-Ga“ el Ottogalli. Dynamic service adaptation for plug and play device interoperability. In Proceedings of the 7th International Conference on Network and Services Management, CNSM '11, pages 46-55, Laxenburg, Austria, Austria, 2011. International Federation for Information Processing.

[13] Ji Eun Kim, G. Boulos, J. Yackovich, T. Barth, C. Beckel, and D. Mosse. Seamless integration of heterogeneous devices and access control in smart homes. pages 206-213, 2012.

[14] B. Kitchenham, R. Pretorius, D. Budgen, Pearl B. O., M. Turner, M. Niazi, and S. Linkman. Systematic literature reviews in software engineering - a tertiary study. Inf. Softw. Technol., 52(8):792-805, August 2010.

[15] J. Krikke. T-engine: Japan's ubiquitous computing architecture is ready for prime time. Pervasive Computing, IEEE, 4(2):4-9, 2005. 
[16] B. Kumar and M. Rahman. Mobility support for Universal Plug and Play (UPnP) devices using session initi-ation protocol (sip). In Consumer Communications and Networking Conference, 2006. CCNC 2006. 3rd IEEE, volume 2, pages 788-792, 2006.

[17] Chin-Feng Lai and Yueh-Min Huang. Context-aware multimedia streaming service for smarthome. In Proceedings of the International Conference on Mobile Technology, Applications, and Systems, Mobility '08, pages 106:1-106:5, New York, NY, USA, 2008.

[18] Jin Mitsugi, Shigeru Yonemura, Hisakazu Hada, and Tat-suya Inaba. Bridging upnp and zigbee with coap: protocol and its performance evaluation. In Proceedings of the workshop on Internet of Things and Service Platforms, IoTSP '11, pages 1:1-1:8, New York, NY, USA, 2011. ACM.

[19] K.J. Patel, S.V. Anand, and S.P. Sumant Kumart. A robust qos framework on android for effective media delivery to dlna enabled home gateway in smart home environment. In Wireless Communications, Networking and Information Security (WCNIS), 2010 IEEE International Conference on, pages 217-222, 2010.

[20] V. Ricquebourg, D. Menga, D. Durand, B. Marhic, L. Delahoche, and C. Loge. The smart home concept : our immediate future. In E-Learning in Industrial Electronics, 2006 1ST IEEE International Conference on, pages 23-28, 2006.

[21] C. Rus, K. Kontola, I.D.D. Curcio, and I. Defee. Mobile tv content to home wlan. Consumer Electronics, IEEE Transactions on, 54(3):1038-1041, 2008.

[22] A. Uribarren, J. Parra, R. Iglesias, J.P. Uribe, and D. Lopez-de Ipina. A middleware platform for application configuration, adaptation and interoperability. In Self-Adaptive and Self-Organizing Systems Workshops, 2008. SASOW 2008. Second IEEE International Confer-ence on, pages 162$167,2008$.

[23] K.I.-K. Wang, W.H. Abdulla, Z. Salcic, N. DeSouza, and S. Ramkumar. Multiagent control system with mobile ubiquitous platform for ambient intelligence. In Intelligent Environments, 2008 IET 4th International Confer-ence on, pages 1-7, 2008.

[24] E.U. Warriach, E. Kaldeli, J. Bresser, A. Lazovik, and M. Aiello. Heterogeneous device discovery framework for the smart homes. In GCC Conference and Exhibition (GCC), 2011 IEEE, pages 637640, 2011.

[25] I.A. Zualkernan, A. R. Al-Ali, M.A. Jabbar, I. Zabalawi, and A. Wasfy. Infopods: Zigbee-based remote information monitoring devices for smart-homes. Consumer Electronics, IEEE Transactions on, 55(3):1221-1226, 2009. 